\title{
Comparison of Test Results for Zika Virus RNA in Urine, Serum, and Saliva Specimens from Persons with Travel-Associated Zika Virus Disease — Florida, 2016
}

\author{
Andrea M. Bingham, $\mathrm{PhD}^{1}$; Marshall Cone, $\mathrm{MPH}^{1}$; Valerie Mock ${ }^{1}$; Lea Heberlein-Larson, $\mathrm{MPH}^{1}$; Danielle Stanek, DVM ${ }^{1}$; \\ Carina Blackmore, DVM, $\mathrm{PhD}^{1}$; Anna Likos, $\mathrm{MD}^{1}$
}

\section{On May 10, 2016, this report was posted as an MMWR Early Release on the MMWR website (http://www.cdc.gov/mmwr).}

In May 2015, Zika virus was reported to be circulating in Brazil. This was the first identified introduction of the virus in the Region of the Americas. Since that time, Zika virus has rapidly spread throughout the region. As of April 20, 2016, the Florida Department of Health Bureau of Public Health Laboratories (BPHL) has tested specimens from 913 persons who met state criteria for Zika virus testing. Among these 913 persons, 91 met confirmed or probable Zika virus disease case criteria and all cases were travel-associated $(1)$. On the basis of previous small case studies reporting real time reverse-transcription polymerase chain reaction (RT-PCR) detection of Zika virus RNA in urine, saliva, and semen (2-G), the Florida Department of Health collected multiple specimen types from persons with suspected Zika virus disease. Test results were evaluated by specimen type and number of days after symptom onset to determine the most sensitive and efficient testing algorithm for acute Zika virus disease. Urine specimens were collected from 70 patients with suspected Zika virus disease from zero to 20 days after symptom onset. Of these, 65 (93\%) tested positive for Zika virus RNA by RT-PCR. Results for $95 \%$ (52/55) of urine specimens collected from persons within 5 days of symptom onset tested positive by RT-PCR; only 56\% (31/55) of serum specimens collected on the same date tested positive by RT-PCR. Results for 82\% (9/11) of urine specimens collected $>5$ days after symptom onset tested positive by RT-PCR; none of the RT-PCR tests for serum specimens were positive. No cases had results that were exclusively positive by RT-PCR testing of saliva. BPHL testing results suggest urine might be the preferred specimen type to identify acute Zika virus disease.

Criteria for Zika virus testing included persons who experienced two or more of the following symptoms: rash, fever, arthralgia or conjunctivitis during or within 2 weeks of return from an area with Zika virus activity, or who had an epidemiologic link to a Zika virus-infected traveler (sexual partner, household member, etc.). RT-PCR was routinely performed on urine, serum, or saliva specimens collected within 21 days of symptom onset. Clinicians were informed that only the serum RT-PCR and antibody tests were to be used for diagnostic purposes. Urine and saliva RT-PCR tests were only used for surveillance purposes.
Serologic testing was performed on all serum specimens included in this analysis. The probable case definition criteria for Zika virus disease, based on serology, required Zika virus-specific IgM antibodies and no dengue virus-specific IgM antibodies detected in serum or cerebrospinal fluid.

Zika virus RT-PCR was performed at BPHL using a laboratorydeveloped test based on a previously published protocol using two RT-PCR targets ( 7 ) (this is not the CDC Trioplex rRTPCR assay authorized for emergency use by the Food and Drug Administration (8)). Specimens were tested in a primary assay, in duplicate in the same run, with a primer and probe set that detects all known genotypes of Zika virus, ZIKV 1086/1162c/1107FAM (later renamed ZIKV 1087/1163c/1108FAM). If detected in at least one of the duplicates, the same extract was tested with a secondary assay, in duplicate in the same run, with a primer and probe set that detects the Asian genotype currently circulating in the Western Hemisphere, ZIKV 4481/4552c/4507cFAM (unpublished Zika real time RT-PCR protocol, RS Lanciotti, Division of Vector-Borne Infectious Diseases, CDC, Fort Collins, Colorado, updated January 14, 2016).

Specimens reported as positive had cycle threshold $(\mathrm{Ct})$ values $\leq 38$ for at least one of the replicates in both the primary and secondary RT-PCR assays. Specimens reported as equivocal had a Ct value $\leq 38$ in the primary assay, but not the secondary assay. For the purpose of this analysis, equivocal specimens were considered as negative. Specimens reported as negative had Ct values $>38$ in the primary assay and were not tested further. Zika virus and dengue virus IgM antibody testing was performed at BPHL using a laboratory-developed IgM antibody capture enzyme-linked immunosorbent assay (MAC-ELISA) based on a CDC flavivirus MAC-ELISA protocol (9). In March 2016, BPHL transitioned to the Food and Drug Administration's Emergency Use Authorization Zika MAC-ELISA developed by CDC (8). Zika virus antigen and positive control material were provided by CDC. A positive/negative $(\mathrm{P} / \mathrm{N})$ ratio was calculated from results of the MAC-ELISA for each specimen tested and was interpreted as the following: $\mathrm{P} / \mathrm{N}$ ratios $<2$ were reported as negative, $\mathrm{P} / \mathrm{N}$ ratios $2-<3$ were reported as equivo$\mathrm{cal}$, and $\mathrm{P} / \mathrm{N}$ ratios $\geq 3$ were reported as presumptive positive, as defined in the emergency use authorization. 
As of April 20,2016, 91 cases of travel-associated Zika virus disease had been reported in Florida. Urine specimens were collected from a total of 70 persons with Zika virus disease, and in 65 (93\%) of the cases, the urine specimen was positive by RT-PCR (Figure). The five specimens that were negative by RT-PCR testing were collected on days 2, 5, 5, 7, and 14 after symptom onset. Viral RNA was detectable in urine as early as the 1 st day of symptoms and as late as 20 days after onset of symptoms. Ten of 12 urine specimens (83\%) collected 7-20 days after symptom onset were positive. Among 62 of the 65 cases with positive urine specimens by RT-PCR testing, both primer and probe sets were positive in duplicate reactions. For two of the three remaining cases, a saliva specimen also tested positive by RT-PCR.

In 66 cases, persons had urine and serum specimens collected on the same day. The majority of these persons were female (64\%), white (77\%), and Hispanic (71\%), with a median age of 46 years (range $=23-76$ years). In two cases, female patients were pregnant. Approximately twice as many persons had RT-PCR positive test results for Zika virus RNA in urine specimens compared with serum specimens, 61 persons (92\%) versus 31 (47\%), respectively. One person had positive test results in serum alone ( 2 days after symptom onset) and 31 persons had positive test results only for urine specimens.
Among the 55 persons with urine and serum specimens collected within the first 5 days of symptom onset, 52 (95\%) had urine specimens that tested positive for Zika virus RNA by RT-PCR testing and 31 (56\%) had serum specimens that tested positive (Table 1). Forty percent (22/55) of the serum specimens had detectable Zika virus IgM antibodies, including two specimens collected 1 day after symptom onset. Among the 11 cases with specimens collected $>5$ days after symptom onset, nine persons $(82 \%)$ had urine specimens that tested positive by RT-PCR; none had serum specimens that tested positive (Table 1).

Three specimen types collected on the same day were available for 53 of the 66 cases and were tested by RT-PCR: $92 \%$ of urine specimens, $81 \%$ of saliva specimens, and $51 \%$ of serum specimens tested positive. Viral RNA was detected in saliva as early as 1 day and as late as 20 days after symptom onset (Table 2). All cases with saliva specimens that tested positive for Zika virus RNA by RT-PCR testing also had at least one other specimen type that tested positive by RT-PCR testing.

Of the 66 serum specimens that also had paired urine specimens, five (8\%) tested positive for both Zika virus RNA and IgM antibody (the five specimens were collected 1, 2, 3, 5, and 5 days after symptom onset) (Table 1). Among the 31 cases in which urine specimens tested positive by RT-PCR, but serum specimens tested negative, Zika virus IgM antibody was detected in serum

FIGURE. Results of RT-PCR testing for Zika virus RNA in urine specimens of 70 persons with travel-associated Zika virus disease, by number of days after onset of symptoms - Florida, 2016*

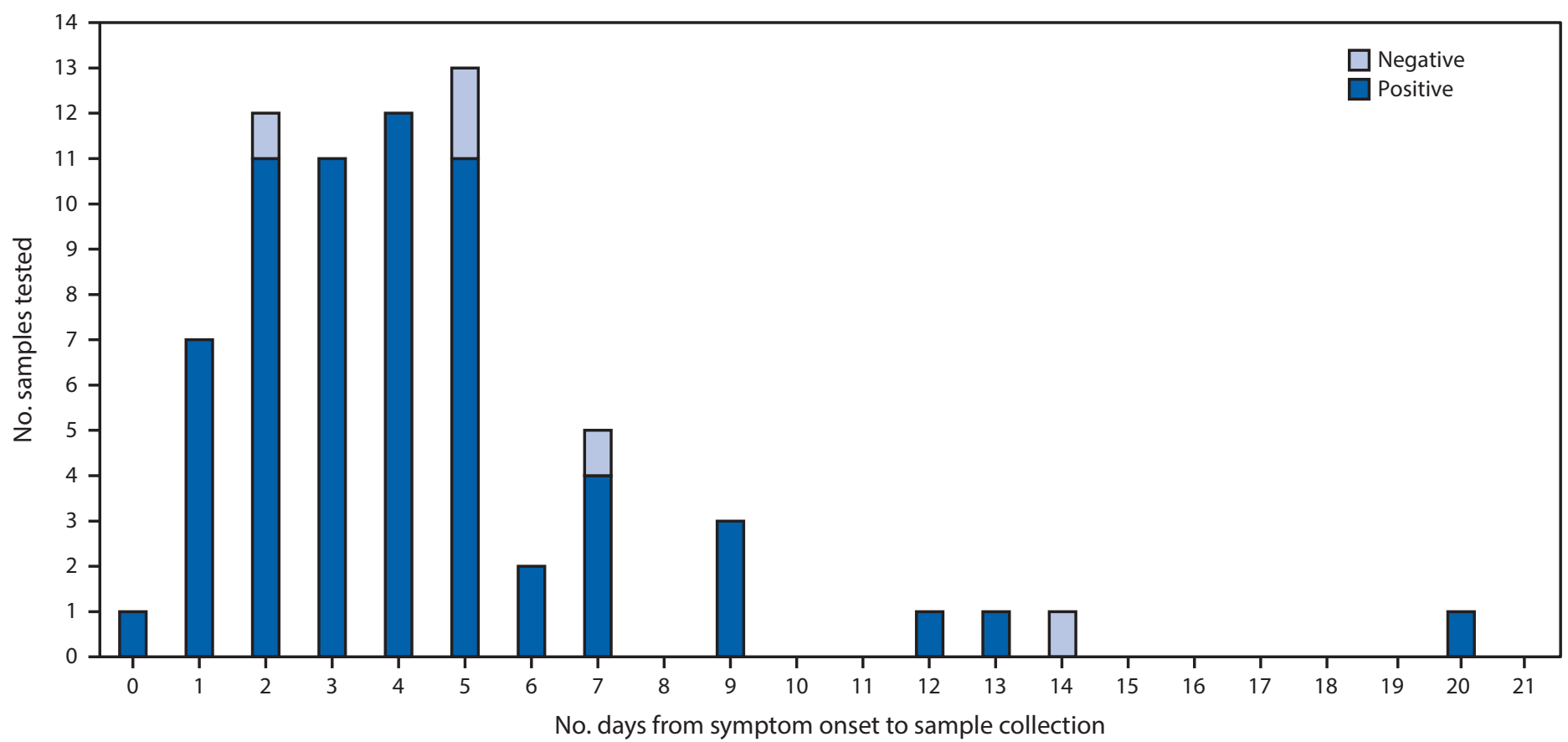

Abbreviation: RT-PCR = reverse transcription-polymerase chain reaction.

* Four persons included in figure did not contribute to the 66 persons with urine and serum specimens collected on the same day; each of these four persons had Zika virus RNA detected in their urine specimens, which were collected on days 3, 7, 12, and 13, respectively. 
TABLE 1. Results of Zika virus IgM antibody testing of serum specimens and RT-PCR testing of serum and urine specimens for Zika virus RNA, by days after symptom onset for 66 persons with travel-associated Zika virus disease — Florida, 2016

\begin{tabular}{lcrr}
\hline Days after onset & $\begin{array}{c}\text { Serum IgM } \\
\text { No. positive/No. tested (\%) }\end{array}$ & $\begin{array}{c}\text { Serum RT-PCR } \\
\text { No. positive/No. tested (\%) }\end{array}$ & $\begin{array}{c}\text { Urine } \\
\text { RT-PCR No. positive/No. tested (\%) }\end{array}$ \\
\hline 0 & $0 / 1(0)$ & $0 / 1(0)$ & $1 / 1(100)$ \\
1 & $2 / 7(29)$ & $6 / 7(85)$ & $7 / 7(100)$ \\
2 & $3 / 12(25)$ & $8 / 12(67)$ & $11 / 12(92)$ \\
3 & $5 / 10(50)$ & $4 / 10(40)$ & $10 / 10(100)$ \\
4 & $3 / 12(25)$ & $8 / 12(67)$ & $12 / 12(100)$ \\
5 & $9 / 13(69)$ & $5 / 13(38)$ & $11 / 13(85)$ \\
6 & $2 / 2(100)$ & $0 / 2(0)$ & $2 / 2(100)$ \\
7 & $4 / 4(100)$ & $0 / 4(0)$ & $3 / 4(75)$ \\
9 & $2 / 3(67)$ & $0 / 3(0)$ & $3 / 3(100)$ \\
14 & $1 / 1(100)$ & $0 / 1(0)$ & $0 / 1(0)$ \\
20 & $1 / 1(100)$ & $0 / 1(0)$ & $1 / 1(100)$ \\
Range of days & & & \\
$0-5$ & $22 / 55(40)$ & $31 / 55(56)^{*}$ & $0 / 9(0)^{*}$ \\
$6-10$ & $8 / 9(89)$ & $0 / 1(0)$ & $52 / 55(95)^{*}$ \\
$11-15$ & $1 / 1(100)$ & $0 / 1(0)$ & $8 / 9(89)^{*}$ \\
$16-20$ & $1 / 1(100)$ & $0 / 1(0)$ & $1 / 1(100)$ \\
\hline
\end{tabular}

Abbreviations: $\lg M=$ immunoglobulin $\mathrm{M} ; \mathrm{RT}-\mathrm{PCR}=$ real time reverse-transcription polymerase chain reaction.

* Statistically significant difference in proportion RT-PCR positive in serum specimens versus urine specimens, by exact McNemar's test ( $0-5$ days, $p<0.001 ; 6-10$ days, $p<0.01)$.

in $23(74 \%)$. Of the remaining eight cases in which neither IgM antibodies nor viral RNA were detected in serum, Zika virus RNA was detected in saliva as well as urine in five cases (the five cases had all three specimens collected on days 2, 3, 4, 5, and 9 after symptom onset, respectively), and in three cases (serum and urine specimens collected days 0,2 , and 3, respectively) saliva specimens were not collected for testing. Overall, Zika virus IgM antibodies were detectable in the serum specimens from $48 \%$ of the 66 cases. Four of the 66 cases had serum and urine specimens that tested negative by RT-PCR testing, but positive (serum specimens only) by IgM antibody testing (specimens collected 5, 5, 7, and 14 days after symptom onset, respectively).

\section{Discussion}

Results of testing conducted at BPHL suggest that urine might be the preferred specimen type to identify acute Zika virus disease. Rates of detection from urine were higher than from serum, even during the first few days after symptom onset and continuing after day five, when no serum specimens tested in this evaluation had detectable RNA. Assays used for diagnostic purposes need to be validated for the specific specimen type being tested. The ability to confirm that a recent illness is caused by Zika virus and not another flavivirus by detection of Zika virus RNA in a clinical specimen is important, given the limitations in interpretation of results from serology testing in persons who have had previous flavivirus infection or vaccination. Among pregnant women, this ability to confirm Zika virus is important because close monitoring during pregnancy is recommended for women with confirmed Zika virus disease. The ease of collection of urine specimens is an additional advantage. This report also demonstrates that saliva specimens (another specimen that is easily obtained) can also yield a higher rate of RNA detection than serum even during the first 5 days; the detection rate in saliva also approaches the detection rate in urine. However, no cases were identified through saliva testing alone.

The findings in this report are subject to at least four limitations. First, eight patients from the group with serum and urine tested by RT-PCR who had RNA detected in their urine specimen but not in their serum specimen did not have Zika virus IgM antibodies detected in their serum to provide an independent confirmation of Zika virus infection. However, five of these eight patients had a saliva specimen available, and all five had viral RNA detected in

\section{Summary}

What is already known about this topic?

Limited data suggest Zika virus is excreted in multiple body fluids, including urine and saliva. Urine and saliva might be appropriate specimens for evaluating Zika virus disease.

What is added by this report?

A comparison of reverse-transcription polymerase chain reaction (RT-PCR) test results for urine and serum specimens from 66 persons with Zika virus disease with both specimens collected on the same date indicated that approximately twice as many urine specimens (61) than serum specimens (31) tested positive. No results from RT-PCR testing of serum specimens were positive $>5$ days after symptom onset; results from testing nine of 11 urine specimens were positive. A further comparison of 53 persons with Zika virus disease with urine, saliva, and serum specimens collected on the same date found positive results from testing in $49(92 \%)$ urine specimens, $43(81 \%)$ saliva specimens, and 27 (51\%) serum specimens.

What are the implications for public health practice?

These results suggest urine might be a useful specimen for identifying acute Zika virus disease. 
Morbidity and Mortality Weekly Report

TABLE 2. Results of RT-PCR testing of urine, saliva, and serum specimens for Zika virus RNA, by days after symptom onset for 53 travel-associated cases of Zika virus disease - Florida, 2016

\begin{tabular}{|c|c|c|c|}
\hline Days after onset & $\begin{array}{c}\text { Urine } \\
\text { No. positive/No. tested (\%) }\end{array}$ & $\begin{array}{l}\text { Saliva } \\
\text { No. positive/No. tested (\%) }\end{array}$ & $\begin{array}{l}\text { Serum } \\
\text { No. positive/No. tested (\%) }\end{array}$ \\
\hline 1 & 7/7 (100) & 7/7 (100) & 6/7 (86) \\
\hline 2 & $9 / 9(100)$ & $9 / 9(100)$ & $6 / 9(67)$ \\
\hline 3 & $9 / 9(100)$ & 8/9 (89) & $4 / 9(44)$ \\
\hline 4 & 9/9 (100) & 8/9 (89) & 7/9 (78) \\
\hline 5 & 10/12 (83) & 9/12 (75) & 4/12 (33) \\
\hline 6 & 1/1 (100) & 0/1 (0) & 0/1 (0) \\
\hline 7 & 2/3 (67) & $0 / 3(0)$ & $0 / 3(0)$ \\
\hline 9 & $1 / 1(100)$ & $1 / 1(100)$ & $0 / 1(0)$ \\
\hline 14 & 0/1 (0) & 0/1 (0) & $0 / 1(0)$ \\
\hline 20 & $1 / 1(100)$ & $1 / 1(100)$ & $0 / 1(0)$ \\
\hline
\end{tabular}

Abbreviation: RT-PCR = real time reverse-transcription polymerase chain reaction.

saliva. The lack of IgM antibodies in some of the cases might be explained by the early timing of the serum collection; Zika virus IgM antibody might be detectable in serum specimens collected as early as 4-5 days after symptom onset, and is usually present by 7 days after symptom onset ( 7 ). However, convalescent serum specimens were not obtained to help confirm Zika virus disease by serology. Second, only five urine specimens came from patients $>7$ days after symptom onset; therefore the RNA detection rate in urine specimens from this period is not well characterized. However, the limited data available demonstrate that testing of some specimens can have positive results as far out as 20 days. Third, date of symptom onset can be difficult to ascertain, particularly in symptoms with mild symptoms. Therefore, the absolute rate of RNA detection for a particular day after symptom onset might be imprecise, but the relative detection rate across specimen types should not be impacted by this limitation. Finally, real-time RT-PCR results should be carefully interpreted to account for the possibility of false-negative and false-positive results, particularly at the lower limits of detection of the assay, when reproducibility is low and results are not confirmed with both primer/probe sets in replicate tests.

\section{Acknowledgments}

Florida Department of Health laboratory and epidemiology personnel.

\footnotetext{
${ }^{1}$ Florida Department of Health.

Corresponding author: Andrea M Bingham, Andrea.Bingham@flhealth.gov, 850-245-4401.
}

\section{References}

1. Council of State and Territorial Epidemiologists. Zika virus disease and congenital Zika virus infection interim case definition and addition to the nationally notifiable disease list. Atlanta, GA: Council of State and Territorial Epidemiologists; 2016. https://www.cste2.org/docs/Zika_ Virus_Disease_and_Congenital_Zika_Virus_Infection_Interim.pdf

2. Gourinat AC, O’Connor O, Calvez E, Goarant C, Dupont-Rouzeyrol M. Detection of Zika virus in urine. Emerg Infect Dis 2015;21:84-6. http://dx.doi.org/10.3201/eid2101.140894

3. Rozé B, Najioullah F, Fergé JL, et al.; GBS Zika Working Group. Zika virus detection in urine from patients with Guillain-Barré syndrome on Martinique, January 2016. Euro Surveill 2016;21:30154. http://dx.doi. org/10.2807/1560-7917.ES.2016.21.9.30154

4. Musso D, Roche C, Nhan TX, Robin E, Teissier A, Cao-Lormeau VM. Detection of Zika virus in saliva. J Clin Virol 2015;68:53-5. http://dx.doi. org/10.1016/j.jcv.2015.04.021

5. Atkinson B, Hearn P, Afrough B, et al. Detection of Zika virus in semen [letter]. Emerg Infect Dis. Epub May 16. http://dx.doi.org/10.3201/eid2205.160107

6. Musso D, Roche C, Robin E, Nhan T, Teissier A, Cao-Lormeau V-M. Potential sexual transmission of Zika virus. Emerg Infect Dis 2015;21:359-61. http://dx.doi.org/10.3201/eid2102.141363

7. Lanciotti RS, Kosoy OL, Laven JJ, et al. Genetic and serologic properties of Zika virus associated with an epidemic, Yap State, Micronesia, 2007. Emerg Infect Dis 2008;14:1232-9. http://dx.doi.org/10.3201/eid1408.080287

8. Food and Drug Administration. Zika virus emergency use authorization. Emergency use authorizations. Silver Spring, MD: US Department of Health and Human Resources, Food and Drug Administration; 2016. http://www.fda.gov/downloads/MedicalDevices/Safety/ EmergencySituations/UCM491592.pdf

9. Martin DA, Muth DA, Brown T, Johnson AJ, Karabatsos N, Roehrig JT. Standardization of immunoglobulin $\mathrm{M}$ capture enzyme-linked immunosorbent assays for routine diagnosis of arboviral infections. J Clin Microbiol 2000;38:1823-6. 